International Journal of Pure and Applied Mathematics

Volume 85 No. 3 2013, 583-592

ISSN: 1311-8080 (printed version); ISSN: 1314-3395 (on-line version)

url: http://www.ijpam.eu

doi: http://dx.doi.org/10.12732/ijpam.v85i3.13

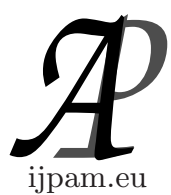

\title{
A COMPARISON OF PERFORMANCE OF RESIDUAL CONTROL CHARTS FOR TREND STATIONARY AR(p) PROCESSES
}

\author{
Yupaporn Areepong \\ Department of Applied Statistics \\ Faculty of Applied Science \\ King Mongkut's University of Technology North Bangkok \\ Bangkok, 10800, THAILAND
}

\begin{abstract}
The main goal of this paper is to implement Statistical Process Control (SPC) with Residual Control Charts. In real life applications, such as in industries or manufacturing, many processes are described by autocorrelated observations. In addition to autocorrelation, some types of industrial processes also exhibit a particular kind of trend behavior. A typical example of process with autocorrelations arises in chemical processes. One of the main characteristics of a control chart are the Average Run Length $\left(A R L_{0}\right)$ (mean of false alarm times) and the Average Delay time $\left(A R L_{1}\right)$ (mean delay of true alarm times). The main parameter observed for a given process is the mean shift. If a process has no mean shift, then the $A R L_{0}$ should be sufficiently large. On the opposite side, when a mean shift occurs the $A R L_{1}$ should be small in order to indicate the mean shift quickly. We compared the performance of the Shewhart - $\bar{x}$, EWMA, and GMA residual control charts for autocorrelation observations with upward/downward linear trend. These comparisons are made for different magnitudes of the mean shift and various levels of autocorrelation.
\end{abstract}

AMS Subject Classification: 60A05

Key Words: Shewhart $-\bar{x}$ chart, exponentially weighted moving average con-

Received: February 27, 2013

(c) 2013 Academic Publications, Ltd. url: www.acadpubl.eu 
trol chart (EWMA), geometric moving average control chart (GMA), average run length (ARL)

\section{Introduction}

Statistical Process Control (SPC) techniques are widely used for monitoring and improving quality of production in many areas of practical applications application, for example, industry and manufacturing, finance and economics, epidemiology and health care, environmental sciences and for many other fields see, Borror et al. [3]; Stoumbos and Reynolds [7]. Traditional control charts are evaluated under the assumption that observations are independent and identically distributed (i.i.d.) and in most cases normally distribution. However, in real applications, many charts have autocorrelated observations. As an example, the processes implemented in a control chart to detect computer intrusions in network systems, or the manufacture of food, chemicals, paper and other wood products. As a results, recently, has been an increasing research interest to study the effect of autocorrelation on the performance of SPC charts.

It has been observed that, the main effect of autocorrelation in the process data within a SPC schemes for a traditional chart the Average Run Length of in-control process may be more shorter than the intended one (see Johnson and Bagshaw [5]). Processes with serial correlation in data also need to be monitored by appropriate control charts.

One of the most widely approach is to fit the autocorrelated data of the observed process into a time series model, such that forecasts of each observation can be made using the previous observations, and apply the fitted data to the residuals of traditional control charts (see e.g. Harris and Ross [6], Lu and Reynolds [4]).

Johnson and Bagshaw [5] derived approximate run length distribution for the Cumulative Sum Control Chart (CUSUM) chart when the process follows a Moving Average model of order one, MA(1), or an Autoregressive Process of order one, AR(1). Harris and Ross [6] discussed the effect of autocorrelation on the performance of Exponentially Weighted Moving Average (EWMA) charts and CUSUM, and found that the Average Run Lengths of these charts were sensitive to the presence of autocorrelation.

Recently, Karaoglan and Bayhan [1] compared performance of Shewhart, CUSUM EWMA and GMA residual control charts for increasing trend of an $\mathrm{AR}(1)$ process. In this paper, we study the residual control charts for $\mathrm{AR}(\mathrm{p})$ process which includes upwards and downward linear trend. We also compare 
the performance of the Shewhart - $\bar{x}$, EWMA, and GMA residual control charts for autocorrelation observations at different magnitudes of the mean shift and various levels of autocorrelation.

\section{Control Charts and Their Properties}

The Average Run Length is an important characteristic for any SPC chart. It is defined as the expectation of the time before the control chart gives a false alarm that an in-control process has run out-of-control $\left(A R L_{0}\right)$. A second important characteristic for a SPC chart is the Average Delay time $\left(A R L_{1}\right)$.

To design and efficient chat the $A R L_{0}$ should be large and the $A R L_{1}$ should be small.

Let $E_{\theta}($.$) denote the expectation that the change-point occurs in a random$ observations generated from a distribution function $F\left(x, \mu, \sigma^{2}\right)$. At one point in time $\theta$ the distribution mean is change from $\mu=\mu_{0}$ to $\mu=\mu_{1}$, where $\theta \leq \infty$. A typical condition imposed on an $A R L_{0}$ is

$$
E_{\theta}(\tau)=T, \quad \theta=\infty \quad(\text { in-control } \quad \text { state }),
$$

where $T$ is given (usually large). For a given distribution function and chart, this condition determine the choices of UCL and LCL and the typical practical constraint is $A R L_{0}=T$.

A typical definition of the $A R L_{1}$ is

$$
A R L_{1}=E_{1}(\tau \mid \tau \geq 1),
$$

means that the change point occurs at $\theta=1$. One could expect that a sequential control chart has a near optimal performance if its $A R L_{1}$ is close to a minimal value. There are many other criteria that could be used for designing optimal SPC charts.

Let $x_{t}$ be autocorrelated observations, then the residual forms a time series model of $x_{t}$ defined as

$$
e_{t}=x_{t}-\hat{x}_{t},
$$

where $\hat{x}_{t}$ is the prediction of $x_{t}$ at time $t$. Residual control charts are construct based on $e_{t}$ depending on the traditional charts used. For a Shewhart residual chart,the $3 \sigma$ upper and lower control limits are defined by

$$
U C L=\bar{e}+3 \sigma_{e}, \quad L C L=\bar{e}-3 \sigma_{e},
$$

where $\bar{e}$ is the center line of the chart with standard deviation $\sigma_{e}$. 
The alarm time for the Shewhart residual chart is given by:

$$
\tau=\inf \left\{t>0: e_{t}>U C L \quad \text { or } \quad e_{t}<L C L\right\}
$$

An EWMA chart is defined by a recursive formula

$$
Y_{t}=(1-\lambda) Y_{t-1}+\lambda e_{t}, \quad t=1,2, \ldots
$$

where $\lambda \in(0,1)$ is a smoothing parameter and $Y_{t}$ is the weighted average between current and previous observations at $t$. The target mean is supposed to be steady and the initial value $e_{t}$ is usually chosen to be the process mean $\mu_{0}$. The upper control limit of the EWMA chart is the following:

$$
U C L=\bar{e}+L \sigma_{e} \sqrt{\frac{\lambda}{2-\lambda}},
$$

and the lower control limit is:

$$
L C L=\bar{e}-L \sigma_{e} \sqrt{\frac{\lambda}{2-\lambda}},
$$

where $L$ is a constant to be chosen. The process will be declared to be in an out-of-control state when $Y_{t}>U C L$ or $Y_{t}<L C L$. The alarm time for the EWMA is then given by

$$
\tau=\inf \left\{t>0: Y_{t}>U C L \quad \text { or } \quad Y_{t}<L C L\right\}
$$

A Geometric Moving Average(GMA) control chart is defined by the following statistics (Karaoglan and Bayhan [2]):

$$
Y_{t}=(1-\lambda) Y_{t-1}+\lambda e_{t}, \quad t=1,2, \ldots
$$

The upper control limit of the GMA chart is the following:

$$
U C L=\bar{e}+L\left(\bar{R} / d_{2}\right)[\lambda /(2-\lambda)]^{1 / 2},
$$

and the lower control limit is:

$$
L C L=\bar{e}-L\left(\bar{R} / d_{2}\right)[\lambda /(2-\lambda)]^{1 / 2}
$$

where $L$ is a constant to be chosen, $\bar{R}$ is an average of range and $d_{2}$ is the mean of the distribution of the relative range which can be referred from statistical 
tables. The process will be declared to be in an out-of-control state when $Y_{t}>U C L$ or $Y_{t}<L C L$. The alarm time for the GMA is then given by

$$
\tau=\inf \left\{t>0: Y_{t}>U C L \quad \text { or } \quad Y_{t}<L C L\right\} .
$$

In general, a $p^{t h}$ - order AR model is defined as follow

$$
z_{t}=c+\phi_{1} z_{t-1}+\phi_{2} z_{t-2}+\ldots+\phi_{p} z_{t-p}+e_{t}
$$

where $c$ is a constant, $\phi_{j}$ is $j^{\text {th }}$ autoregressive parameters and $e_{t}$ is the error term of time t. There are specific restriction for different cases:

$$
\begin{gathered}
\text { when } \quad p=1, \quad-1<\phi_{1}<1 \quad \text { and } \\
\text { when } p=2, \quad-1<\phi_{2}<1, \quad \phi_{1}+\phi_{2}<1, \quad \phi_{2}-\phi_{1}<1 .
\end{gathered}
$$

Let $Z_{t}$ be a linear trend represented by:

$$
Z_{t}=z_{t}+d t
$$

where $d$ is the trend slope in term of $t$.

\section{Comparison of Performance of Control Charts}

In this section, the numerical results for $A R L_{0}$ and $A R L_{1}$ for Shewhart, EWMA and GMA residual charts are presented. Table 1 shows a comparison of ARL for $\mathrm{AR}(1)$ process with values obtained via simulation.

The parameter values for Shewhart, EWMA and GMA residual charts were chosen by setting the desired $A R L_{0}=370, \phi_{1}=0.75$, the value of the in-control parameter $\mu=0$ and the shift parameter $\delta \subseteq[0.5 ; 4]$.

For the EWMA and GMA procedures, the parameter values $\lambda=0.05$ and $U C L=2.504$ and 2.613 respectively. The results shown that for $d=0.5$, the EWMA residual chart have a better performance than the other charts when the value of the parameter $\delta$ had a small and moderate shifts.

For example, when $\delta=1$ the EWMA chart shows the best performance because it gives the minimum $\mathrm{AD}$ value (17.736). However, the performance of the Shewhart residual chart is superior to EWMA and GMA charts when $\delta>2$, i.e., when the shift is large. The results for $d=-0.5$ are in good agreement with the results for the results $d=0.5$. 
Table 1: Comparison of ARL for AR(1) on Shewhart EWMA and GMA residual charts when given $A R L_{0}=370$ and $\phi_{1}=0.75$.

\begin{tabular}{|c|c|c|c|c|}
\hline \multirow{1}{*}{$d$} & \multirow{6}{*}{$\delta$} & \multicolumn{3}{|c|}{ ARL } \\
\cline { 3 - 5 } & 0 & SHEWHART & EWMA & GMA \\
\hline & 0.5 & 369.459 & 371.336 & 371.222 \\
& 1 & 58.875 & $17.736^{*}$ & 17.740 \\
& 1.5 & 25.941 & $13.583^{*}$ & 13.589 \\
& 2 & 15.553 & $11.961^{*}$ & 11.966 \\
& 2.5 & $11.034^{*}$ & 11.324 & 11.323 \\
& 3 & $8.262^{*}$ & 11.155 & 11.159 \\
& 3.5 & $5.416^{*}$ & 9.468 & 9.582 \\
& 4 & $3.128^{*}$ & 6.412 & 6.658 \\
\hline-0.5 & 0 & 369.899 & 370.650 & 371.398 \\
& 0.5 & 166.136 & $27.438^{*}$ & 27.460 \\
& 1 & 47.349 & $10.259^{*}$ & 10.272 \\
& 1.5 & 14.205 & $5.951^{*}$ & 5.954 \\
& 2 & 4.719 & $4.192^{*}$ & 4.194 \\
& 2.5 & $2.089^{*}$ & 3.278 & 3.279 \\
& 3 & $1.279^{*}$ & 2.716 & 2.719 \\
& 3.5 & $1.254 *$ & 2.584 & 2.596 \\
& 4 & $1.106^{*}$ & 2.109 & 2.201 \\
\hline
\end{tabular}

Table 2 gives a comparison of ARL for $\mathrm{AR}(1)$ process. The parameter values for Shewhart, EWMA and GMA residual charts were chosen by setting the desired $A R L_{0}=370, \phi_{1}=-0.5$, results for $A R L_{0}$ and $A R L_{1}$ for Shewhart, EWMA and GMA residual charts presented in a similar manner to Table 1.

Table 3 shows a comparison of ARL for $\mathrm{AR}(2)$ process. The parameter values for Shewhart, EWMA and GMA residual charts were chosen by setting the desired $A R L_{0}=370, \phi_{1}=0.75, \phi_{2}=-0.5$, the value of the in-control parameter $\mu=0$ and the shift parameter $\delta \subseteq[0.5 ; 4]$.

For the EWMA and GMA procedures, the parameter values $\lambda=0.05$ and $U C L=2.204$ and 2.137 respectively. The results shown that for $d=0.5$, the EWMA residual chart performs better than the other charts when the value of the parameter $\delta$ had a small and moderate shifts.

For example, when $\delta=0.5$ the EWMA chart shows the best performance 
Table 2: Comparison of ARL for AR(1) on Shewhart EWMA and GMA residual charts when given $A R L_{0}=370$ and $\phi_{1}=-0.5$.

\begin{tabular}{|c|c|c|c|c|}
\hline \multirow{1}{*}{$d$} & & \multicolumn{3}{|c|}{ ARL } \\
\cline { 3 - 5 } & $\delta$ & SHEWHART & EWMA & GMA \\
\hline 0.5 & 0 & 370.002 & 369.073 & 369.532 \\
& 0.5 & 83.315 & $15.467^{*}$ & 15.469 \\
& 1 & 16.349 & $7.248^{*}$ & 7.249 \\
& 1.5 & 5.312 & $5.181^{*}$ & 5.183 \\
& 2 & 2.742 & $4.258^{*}$ & 7.259 \\
& 2.5 & $2.137^{*}$ & 3.755 & 3.757 \\
& 3 & $1.847^{*}$ & 2.747 & 2.749 \\
& 3.5 & $1.467^{*}$ & 2.516 & 2.524 \\
& 4 & $1.108^{*}$ & 2.018 & 2.124 \\
\hline-0.5 & 0 & 371.817 & 370.777 & 370.221 \\
& 0.5 & 85.748 & $16.132^{*}$ & 16.151 \\
& 1 & 18.016 & $7.709^{*}$ & 7.713 \\
& 1.5 & 5.613 & $5.493^{*}$ & 5.496 \\
& 2 & $2.794^{*}$ & 4.471 & 4.472 \\
& 2.5 & $2.166^{*}$ & 3.912 & 3.916 \\
& 3 & $1.736^{*}$ & 2.487 & 2.487 \\
& 3.5 & $1.358^{*}$ & 2.195 & 2.198 \\
& 4 & $1.193^{*}$ & 1.872 & 1.948 \\
\hline
\end{tabular}

because the $\mathrm{AD}$ have a minimal value (39.773). However, the performance of the Shewhart residual chart is superior to EWMA and GMA charts when $\delta>2$, i.e., when the shift is large. The results for $d=-0.5$ are in good agreement with the results for the results $d=0.5$.

In Table 4, the comparison of ARL for an $\mathrm{AR}(2)$ process are presented. The parameter values for Shewhart, EWMA and GMA residual charts were chosen by setting the desired $A R L_{0}=370, \phi_{1}=0.5, \phi_{2}=0.25$, the results for $d=0.5$ shown that the EWMA residual chart have a best performance for small and moderate shifts but Shewhart residual chart perform better than EWMA and GMA charts when $\delta>3$. For $d=-0.5$, the performance of the EWMA residual chart is superior to Shewhart and GMA charts when $0.5 \leq \delta \leq 2$ but the performance of the Shewhart residual chart is superior to EWMA and GMA charts when $\delta>2$. 
Table 3: Comparison of ARL for AR(2) on Shewhart EWMA and GMA residual charts when given $A R L_{0}=370, \phi_{1}=0.75$ and $\phi_{2}=-0.5$.

\begin{tabular}{|c|c|c|c|c|}
\hline \multirow{1}{*}{$d$} & \multirow{6}{*}{$\delta$} & \multicolumn{3}{|c|}{ ARL } \\
\cline { 3 - 5 } & & SHEWHART & EWMA & GMA \\
\hline 0.5 & 0 & 370.609 & 369.508 & 370.983 \\
& 0.5 & 207.821 & $39.773^{*}$ & 39.874 \\
& 1 & 77.479 & $15.934^{*}$ & 15.942 \\
& 1.5 & 29.164 & $9.756^{*}$ & 9.760 \\
& 2 & 11.066 & $6.941^{*}$ & 6.945 \\
& 2.5 & $4.370^{*}$ & 5.270 & 5.272 \\
& 3 & $2.487^{*}$ & 4.171 & 4.171 \\
& 3.5 & $2.295^{*}$ & 3.465 & 3.601 \\
\hline-0.5 & 0 & $1.954^{*}$ & 2.841 & 2.934 \\
& 0.5 & 370.757 & 370.544 & 370.291 \\
& 1 & 188.674 & $34.929^{*}$ & 34.902 \\
& 1.5 & 18.051 & $13.148^{*}$ & 13.188 \\
& 2 & 5.613 & $7.902^{*}$ & 7.903 \\
& 2.5 & $2.711^{*}$ & $5.535^{*}$ & 5.536 \\
& 3 & $2.139^{*}$ & 3.233 & 4.253 \\
& 3.5 & $1.782 *$ & 3.185 & 3.521 \\
& 4 & $1.428^{*}$ & 2.683 & 2.142 \\
\hline
\end{tabular}

\section{Conclusion}

We compared the effectiveness of the Shewhart - $\bar{x}$, EWMA, and GMA residual control charts for autocorrelation observations with upward and downward linear trend. The comparison of the control charts was based on the Average Run Length $\left(A R L_{0}\right)$ and the Average Delay time $\left(A R L_{1}\right)$ criteria. For all orders of an $A R(p)$ process analyzed in this research, we shown that the performance of EWMA charts is superior to the Shewhart $-\bar{x}$ and GMA residual charts for small shifts, however the performance of Shewhart - $\bar{x}$ residual chart is superior to EWMA and GMA residual charts for large shifts. 
Table 4: Comparison of ARL for AR(2) on Shewhart EWMA and GMA residual charts when given $A R L_{0}=370, \phi_{1}=0.5$ and $\phi_{2}=0.25$.

\begin{tabular}{|c|c|c|c|c|}
\hline \multirow{1}{*}{$d$} & & \multicolumn{3}{|c|}{ ARL } \\
\cline { 3 - 5 } & $\delta$ & SHEWHART & EWMA & GMA \\
\hline 0.5 & 0 & 370.499 & 370.068 & 370.686 \\
& 0.5 & 166.291 & $33.590^{*}$ & 33.784 \\
& 1 & 54.400 & $17.441^{*}$ & 17.831 \\
& 1.5 & 25.126 & $13.676^{*}$ & 13.774 \\
& 2 & 15.949 & $12.012^{*}$ & 12.014 \\
& 2.5 & 12.473 & $11.117^{*}$ & 11.118 \\
& 3 & 10.717 & $10.701^{*}$ & 10.703 \\
& 3.5 & $8.542^{*}$ & 9.521 & 9.698 \\
& 4 & $6.294 *$ & 8.293 & 8.649 \\
\hline-0.5 & 0 & 371.929 & 370.795 & 370.006 \\
& 0.5 & 160.710 & $26.162^{*}$ & 26.347 \\
& 1 & 43.910 & $10.655^{*}$ & 10.662 \\
& 1.5 & 13.773 & $6.780^{*}$ & 6.782 \\
& 2 & 5.401 & $5.170^{*}$ & 5.184 \\
& 2.5 & $3.099^{*}$ & 4.321 & 4.322 \\
& 3 & $2.384^{*}$ & 3.796 & 3.798 \\
& 3.5 & $2.190^{*}$ & 2.691 & 2.721 \\
& 4 & $1.755^{*}$ & 2.019 & 2.251 \\
\hline
\end{tabular}

\section{Acknowledgments}

The author would like to give special thanks to Dr.Gabriel Mititelu for careful proof-reading and suggestions. The author would also like to express her gratitude to Faculty of Science, King Mongkut's University of Technology, North Bangkok, Thailand for a supporting research grant of No: 5645105.

\section{References}

[1] A.D. Karaoglan and G.M. Bayhan, Performance Comparison of Residual Control Charts for Trend Stationary First Order Autoregressive Processes, Gazi University Journal of Science, 24 (2011), 329-339. 
[2] A.D. Karaoglan and G.M. Bayhan, ARL performance of residual control charts for trend AR(1) process: A case study on peroxide values of stored vegetable oil, Scientific Research and Essays, 7 (2012), 1405-1414.

[3] C.M. Borror, D.C. Montgomery and G.C. Runger, Robustness of the EWMA Control Chart to Non-normality, Journal of Quality Technology, 31 (1999), 309-316.

[4] C.W. Lu and M.R. Reynolds, EWMA control charts for monitoring the mean of autocorrelated processes, J Qual Technol, 31 (1999), 166-188.

[5] R. A. Johnson and M. Bagshaw, The effect of serial correlation on the performance of CUSUM tests, Technometrics, 16 (1974), 103-112.

[6] T. J. Harris and W. H. Ross, Statistical process control procedures for correlated observations, Canadian Journal of Chemical Engineering, 69, (1991), 48-57.

[7] Z.G. Stoumbos and M.R. Reysnolds, Robustness to non-normality and autocorrelation of individuals control charts for monitoring the process mean and variance, Journal of Statistical Computation and Simulation, 66 (2000), 145-187. 